\title{
"Il tempo non guarisce le ferite": un laboratorio didattico su guerra, occupazione e Resistenza a Collecchio (1943-45)
}

L'articolo è una panoramica dei risultati di un laboratorio storico-didattico su guerra, occupazione e Resistenza a Collecchio dal 1943 al 1945. Il progetto è culminato nella pubblicazione di un opuscolo da parte degli studenti che hanno partecipato, intitolato "Il tempo non guarisce le ferite”.

The article is an overview of the results of a laboratory on war, occupation and Resistance in Collecchio (Italy) from 1943 to 1945. The project culminated in the publication of a little pamphlet written by the students who participated, titled "Time does not heal all wounds".

\section{Introduzione}

La pubblicazione Il tempo non guarisce le ferite nasce da un'esperienza laboratoriale di due classi terze della scuola secondaria di primo grado "D. Galaverna" di Collecchio, in collaborazione con l'Istituto storico della Resistenza e dell'età contemporanea di Parma. Nell'ambito del progetto Percorsi del Novecento, che ogni anno mette a confronto studenti, insegnanti e storici con lo scopo di realizzare un vero e proprio viaggio nella scuola come ricerca e come laboratorio, le insegnanti Fiorita Canali e Alessandra Colli hanno scelto di concentrarsi su un periodo storico di transizione e attraversato da non poche complessità e ambiguità: quello compreso tra l'8 settembre 1943 e la Liberazione, a Collecchio, a Parma e nella provincia di Parma. In quei lunghi mesi il conflitto, in Italia, fu percepito 
con ulteriore intensità rispetto al triennio 1940-1943, in quanto non riguardava più situazioni tutto sommato avvertite come lontane - nonostante il significativo e doloroso legame con i fatti militari costituito dai segnali, pur intermittenti, di mariti, genitori, figli, amici che svolgevano il servizio militare sui fronti - ma diveniva una realtà visibile e tangibile, che investiva direttamente il territorio nazionale, con due eserciti stranieri che si affrontavano sulla penisola e l'occupazione nazifascista nell'Italia centro-settentrionale. Proprio per questo motivo il titolo scelto per il laboratorio è stato La guerra in casa. Sulla base di questa impostazione, si è deciso di approfondire le questioni legate alla vita quotidiana, miscelando documentazione istituzionale relativa alla storia locale e fonti e narrazioni delle persone comuni. Come reagirono i collecchiesi e i parmensi alla quotidianità della guerra $\mathrm{e}$ dell'occupazione? Come vi si contrapposero? Come si difesero e reagirono a violenze, soprusi, repressione, privazione delle libertà fondamentali? Ancora, come sopperirono alla difficoltà nel reperimento di cibo o oggetti di uso comune, e quale fu l'effetto dei bombardamenti, dal punto di vista materiale ma anche psicologico? Ragazzi e ragazze hanno cercato di rispondere attivamente a queste domande, sollecitati non da semplici lezioni frontali, ma da un lavoro pratico basato sull'analisi e la discussione delle fonti. Sono stati utilizzati da un lato documenti tradizionali, su base cartacea, provenienti in gran parte dall'Archivio dell'Istituto (carte d'archivio, volantini, stampa: dalle comunicazioni della Prefettura a quelle degli occupanti tedeschi, dai fogli in ciclostile prodotti dai partigiani in città alle immagini della Resistenza in montagna); dall'altro materiale audiovisivo e nuovi strumenti multimediali. A questo proposito metodologicamente funzionale e accattivante è stato l'utilizzo di ResistenzamAPPe, applicazione per tablet e smartphone sviluppata nel 2014 dalla rete degli Istituti storici dell'Emilia Romagna insieme alla piattaforma editoriale Bradypus. Gli studenti si sono mostrati interessati, oltre che alle fotografie, ai documenti e ai filmati riguardanti la città di Parma contenuti all'interno dell'app, anche al particolare tipo di supporto, innovativo e prossimo alla loro esperienza di comunicazione quotidiana.

Il laboratorio si è svolto in due incontri di due ore ciascuno. Tra un appuntamento e l'altro ogni studente è stato sollecitato a riflettere su quanto appreso e a produrre un pensiero personale, in qualsiasi forma, sugli argomenti della guerra, dell'occupazione, della Resistenza e della loro percezione in una comunità di uomini e donne. Da questo lavoro sono scaturiti disegni, poesie, raccolte di testimonianze di amici, parenti, familiari, testi di varia natura che gli stessi ragazzi hanno voluto raccogliere in un piccolo quaderno. Il loro operato ha infine avuto un momento di restituzione pubblica, in quanto è stato presentato ufficialmente alle celebrazioni del 2 giugno 2016 del Comune di Collecchio. 


\section{Cosa resta della guerra?}

Nello stesso modo in cui sul complesso della Pilotta a Parma rimane, ben visibile, quella profonda lesione provocata dalle bombe degli Angloamericani, agli occhi di ragazzi e ragazze la lacerazione è chiaramente avvertibile nel racconto di un nonno o di un bisnonno perseguitato dalle brutture del conflitto, lontane più di settant'anni ma ancora insepolte. Per quanto il tempo trascorra, le ferite non si riemarginano mai completa-

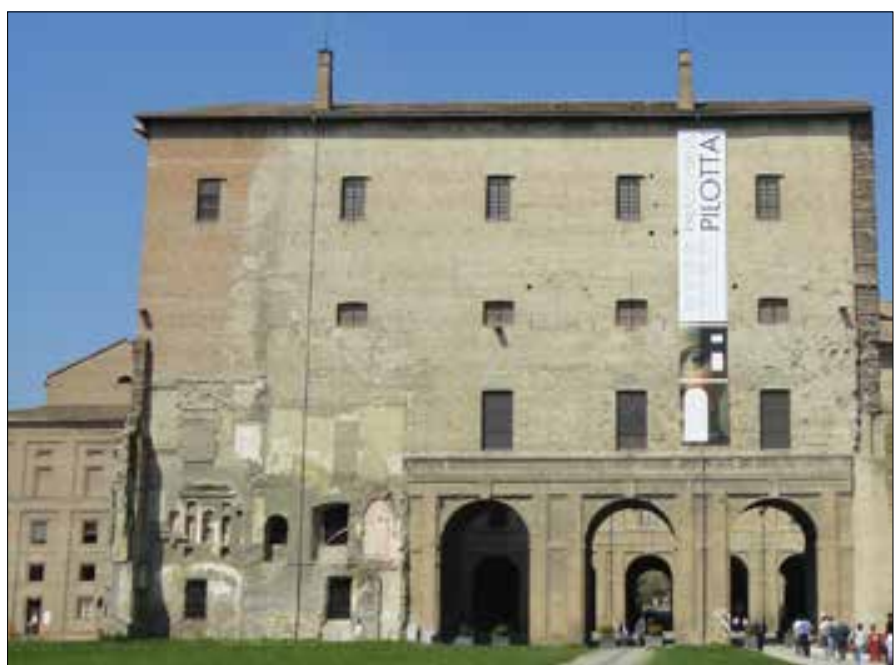

Palazzo della Pilotta

mente: permangono invece nelle vite degli uomini, nei traumi personali e collettivi, negli stessi luoghi in cui viviamo oggi, che portano il segno indelebile di quella storia distruttiva. Questo è il messaggio che gli studenti hanno voluto trasmettere, con la scelta di intitolare il quaderno Il tempo non guarisce le ferite.

L'Istituto ha sostenuto con forza questa decisione, in quanto in essa è stata ravvisata una chiave di lettura genuina, empatica, stimolata evidentemente sia dal contatto diretto con le testimonianze dei familiari, sia dalle considerazioni fatte in classe: ma che a nostro avviso affonda le sue radici nella comprensione profonda, da parte degli studenti, del fattore umano della guerra pur nella sua evidente disumanità, e nella consapevolezza che certe tracce non si cancellano con il tempo.

[...] Un altro orrore, che ricordo, è quello che tutte le sere passava un aereo militare di nome "Pippo": se trovava una luce accesa lui immediatamente bombardava, dunque eravamo obbligati a stare in casa al buio e nascosti o sotto il tavolo o sotto il letto.» La mia bisnonna mi ha raccontato tutto questo piangendo e provando ancora terrore, nonostante sia passato molto tempo. [Giulia Corsi]

L'ultimo avvenimento che ha ricordato sono i tedeschi e i partigiani che ogni giorno passavano per Valbona [Berceto, ndA], dato che il paese era un luogo di passag- 
gio per raggiungere le città in pianura. Mia nonna nasconde ancora le sensazioni, la tristezza, la paura che ha provato in quei giorni, aspetti che si riescono a percepire guardandola mentre mi parlava di ciò. [Alberto Molinari]

La riflessione, tuttavia, non è rimasta circoscritta alla Seconda guerra mondiale nel collecchiese, ma si è allargata ai conflitti attuali, localizzati in diverse zone del mondo. Immediata, ad esempio, è stata l'identificazione sia con i coetanei di allora che con quelli di oggi: le foto dei bambini di Aleppo in Siria, ben radicate nella memoria recente della maggior parte degli studenti, si sono quasi sovrapposte, sul piano emotivo, alle immagini e ai racconti sui ragazzini del '43; nonostante la consapevolezza della diversità dei contesti, chiara è stata la percezione della tragicità di entrambe le condizioni. Ecco come Elisa Barbieri, in una lettera indirizzata a una partigiana, effettua questa operazione, esprimendo un senso di gratitudine per una forza e una determinazione servite allora per resistere contro gli occupanti tedeschi, ma - e il pensiero corre spontaneamente alle guerrigliere curde - indirizzato anche alle combattenti di oggi:

Tu hai deciso di collaborare per la Liberazione. Il bello di questo movimento è questo carattere collettivo, nato dall'iniziativa spontanea di molte e non dalla volontà di poche, senza bisogno di essere creature eccezionali e da qualsiasi strato della società. In Italia adesso non c'è bisogno di fermare la guerra che abbiamo in casa, per ora fortunatamente no, ma fuori sì. Molte volte ci comportiamo come se al mondo non esistesse che l'Italia. Bisogna cercare di uscire fuori dalla propria "patria" e guardare ciò che succede lontano da noi. In questo momento c'è la guerra, si sta combattendo ad esempio contro l'Isis, e in altri paesi dove i bombardamenti sono atti quotidiani, dove avere la "guerra in casa" è normale. [Elisa Barbieri]

È inquietante vedere documenti, oggetti ed armi che provengono dal passato, dalla nostra storia...oggi, quando si parla di guerre e di attentati anche negli altri paesi, mi viene da pensare quanto sia inutile perché porta solo morte... [Alessandro Barbieri]

\section{Persone comuni, vita quotidiana}

Gli elaborati finali sono il frutto sia del percorso di ricerca effettuato in classe, sia delle interviste dei ragazzi a loro familiari o conoscenti. Le nozioni preliminari necessarie per approdare alla fase delle testimonianze sono state acquisite attraverso un coinvolgimento attivo nell'analisi delle fonti dell'epoca, e affrontando diversi temi. In primo luogo si è parlato dell'occupazione del territorio, a maggior ragione in quanto tutta la fascia pedemontana - ricca di vie di collegamento, tra 
cui la statale della Cisa e la ferrovia Parma-La Spezia - era risultata, fin dai giorni immediatamente successivi all'armistizio, fondamentale nella strategia tedesca di contenimento degli Alleati a sud dell'Appennino tosco-emiliano. Di conseguenza Collecchio e le altre località situate lungo le vie di collegamento avevano conosciuto un insediamento particolarmente massiccio di reparti tedeschi o di presidi italiani della Repubblica sociale [Del Sante 1995, 85]. In questo caso, ad esempio, la presenza degli occupanti, spesso dislocati nelle grandi ville del territorio, è stata seguita e illustrata attraverso mappe che ne mettono in rilievo posizione e diffusione.

Ancora, si è trattato della questione dell'approvvigionamento, del sistema delle carte annonarie ma anche del mercato nero, pratica diffusa a Collecchio come in tutta Italia anche prima del 1943. Si è sottolineato, però, come il collocamento nelle campagne abbia costituito in certo modo un beneficio per i collecchiesi, che potevano provvedere più facilmente degli abitanti della città al proprio sostentamento attraverso l'agricoltura e l'allevamento. Particolare attenzione è stata dedicata ai bombardamenti, sia dal punto di vista delle ragioni strategiche degli Alleati, sia da quello della popolazione civile, che si ingegnava per tentare di prevenirne disastrose conseguenze (attraverso le pratiche del coprifuoco o dell'oscuramento) o per mettersi in salvo, in gran parte nelle cantine delle case, poiché a Collecchio non esistevano veri e propri rifugi pubblici.

Mio nonno si ricorda anche di quando suonava l'allarme antiaereo, per segnalare l'arrivo di un bombardiere, e tutta la gente doveva nascondersi per proteggersi dalle bombe, chi nei fossi e chi nelle cantine. [Roberto Percalli]

Non secondarie sono state poi la dimensione e le manifestazioni della violenza, dalle sofferenze e privazioni vissute nel paese alle esperienze di deportazione. Tutti questi elementi sono ricorsi nelle narrazioni raccolte dai ragazzi, che a seconda della loro provenienza hanno raccontato episodi differenti:

Il mio bisnonno [...] nacque nel 1912 a Milano. [...] Dopo l'8 settembre del 1943 lui, come altri soldati italiani, divenne nemico dei tedeschi e proprio da loro fu fatto prigioniero e incarcerato in attesa della deportazione in Germania. [...] Durante il viaggio per la Germania, si fermarono in diverse stazioni e fu proprio durante una di queste soste che [...] vide l'occasione per fuggire e, finalmente, tornare a casa. Quindi si impadronì del berretto di un ferroviere, se lo mise in testa e scese dal treno attraverso un'apertura nel vagone che serviva per il ricambio dell'aria. Grazie al cappello che portava, nessuno sospettò di lui e fece perdere rapidamente le sue tracce. Lì iniziò la lunga marcia per tornare a casa dalla sua famiglia. [Edoardo Dionisio]

Aldo tornò a casa (al Torrione, nel parmense) nel giugno '46, a guerra già finita da 
un anno. Lui era molto spaventato della situazione famigliare che avrebbe potuto trovare, aveva il terrore di essere rimasto solo. Durante il viaggio per tornare a casa sua, passava per i campi come se fosse un ricercato, e se qualcuno passava si nascondeva nei fossi. Arrivato dalla famiglia, che era al completo, tranne per il padre, che morì, per lui la reintegrazione fu molto difficile. Inizialmente parlava di rado e mangiava in piedi, come se fosse ancora un prigioniero. [Pietro Brunazzi]

Lo zio di mio nonno fu deportato in Germania in un campo: durante la notte andava insieme ai suoi compagni in cerca di topi da mangiare per evitare di morire di fame. [Matteo Mora]

La vita nel campo era durissima: il cibo fornito era poca "brodaglia", e [mio nonno] veniva insultato e minacciato. [Nicole Miodini]

«Verso la fine della guerra, l'altra mia sorella Carla, che aveva sui 12-13 anni, andando in giro con i suoi amici, un giorno trovarono una bomba inesplosa, vicino al fiume: un suo amico la maneggiò, ma finì per esplodere. Lui morì e mia sorella rimase molto ferita.». Rammentando questi fatti, ho visto in mia nonna molta tristezza, ma è stata felice di sapere che noi giovani ci interessiamo a queste vicende, che non vanno dimenticate. [Luna Albano]

C'è anche chi ha provato a immedesimarsi nelle vicende della guerra e ad immaginare il dolore provocato dalla perdita di una persona cara:

Quell'uomo era mio padre. Ma non poteva esserlo. Mio padre non era morto! Era solo andato in guerra. Iniziai a piangere e corsi ad abbracciarlo. Non cambiai posizione per molto tempo fino a che mia madre non mi disse di lasciare riposare il papà. Allora gli augurai buona notte e andai in camera a dormire. Mi ritrovai a fissare il soffitto e a pensare a che cosa era appena accaduto. Tutto quello che potei concludere con la mia mente troppo evoluta per un ragazzino di sette anni fu che la guerra aveva ucciso mio padre.

Per ragazze e ragazzi è risultato subito semplice effettuare connessioni tra dati che prima avevano letto solo sui libri e le narrazioni familiari, personali. Il legame emotivo con gli avvenimenti, il coinvolgimento diretto ha determinato una più immediata e sincera comprensione dei fatti manualistici. Questo passaggio è stato non solo utile, ma necessario per comprendere più a fondo il significato della guerra, dell'occupazione, della Resistenza: ha alimentato l'idea secondo la quale la storia non è solo sistema, ma narrazioni delle persone all'interno di un sistema. Gli studenti hanno inoltre compreso a fondo le differenze tra la vita quotidiana di allora e quella odierna, riflettendo sulle radici profonde degli agi del contesto storico-sociale attuale, pacificato e democratico. Sopperire ai propri bisogni, consumare prodotti addirittura superflui rispetto alle proprie reali necessità, esprimere liberamente le idee, per quanto diverse tra loro esse siano: tutte 
queste azioni sono state conquistate a seguito di un percorso disseminato anche da travagli e sofferenze.

Mentre mio padre mi raccontava questa storia, pensavo a tutto quello che quelle persone hanno dovuto subire; mi rendo conto di quanto noi siamo stati fortunati a non aver provato tutto questo. [Valeria Polidori]

Per noi, ragazzi del XXI secolo, i concetti di libertà e di Resistenza sono lontani ma, da parole astratte, queste si sono fatte concrete ed è importante conoscere le persone che hanno lottato per combattere l'oppressione che nega il libero pensiero. [Nicolò Gabella]

Inizialmente ero convinta che la guerra fosse stata uno sfondo piuttosto sfumato e vago e che a rimetterci la vita fossero stati solo i soldati. Invece mi sbagliavo di grosso: la vita dei civili fu letteralmente bloccata e sostituita dagli orrori della guerra. [Elena Marenghi]

Le due classi hanno dunque colto e fatto emergere nella loro produzione la dissonanza tra il privilegio legato a questa condizione e l'esperienza vissuta da nonni e nonne, bisnonni o bisnonne poco più di settant'anni fa. Molti di questi ultimi erano ancora molto piccoli quando scoppiò la Seconda guerra mondiale; lontani dalla consapevolezza dell' età adulta, di quegli anni hanno rammentato soprattutto le privazioni, la scomparsa improvvisa di amici, parenti e familiari, la fame costante. Di fronte a quello stato di necessità spesso - i giovani intervistatori lo hanno messo in rilievo a più riprese nel quaderno, non senza orgoglio - la reazione consisteva in una sorta di adattamento creativo:

Mio nonno per giocare, nel poco tempo libero, riciclava gli stracci per fare dei palloni. Aveva anche costruito degli sci di legno con dei pezzi riciclati. [Guglielmo Boi]

Mia madre in cucina, dalla credenza aveva tolto le stoviglie, il vasellame, le bottiglie, e i bicchieri allineandoli con cura sul pavimento a ridosso delle pareti perimetrali. Si diceva che così facendo si potevano salvare dallo spostamento d'aria provocato dalle esplosioni. [Alessandro Dodi]

Mio nonno Adriano [...] mi ha raccontato di quando usava la seta dei paracaduti per farsi dei vestiti, oppure ricorda quando curava e nutriva dei conigli che dopo mangiava. [Pietro Ughini] 


\section{II ricordo della Resistenza e della Liberazione}

Molti studenti hanno poi riportato testimonianze legate alle esperienze resistenziali, a Collecchio ma anche in altre zone del Parmense:

Il mio bisnonno, Giovanni Savi, all'età di 20 anni entrò nella resistenza partigiana e combatté per liberare Collecchio dalla morsa nazista. Collecchio, infatti, era in mano alle truppe naziste e alcuni aerei da guerra statunitensi bombardarono la polveriera posta nei pressi di Pontescodogna. La sua squadra d'azione era garibaldina e il suo nome di riconoscimento era "il Bimbo". [Pablo Barbato]

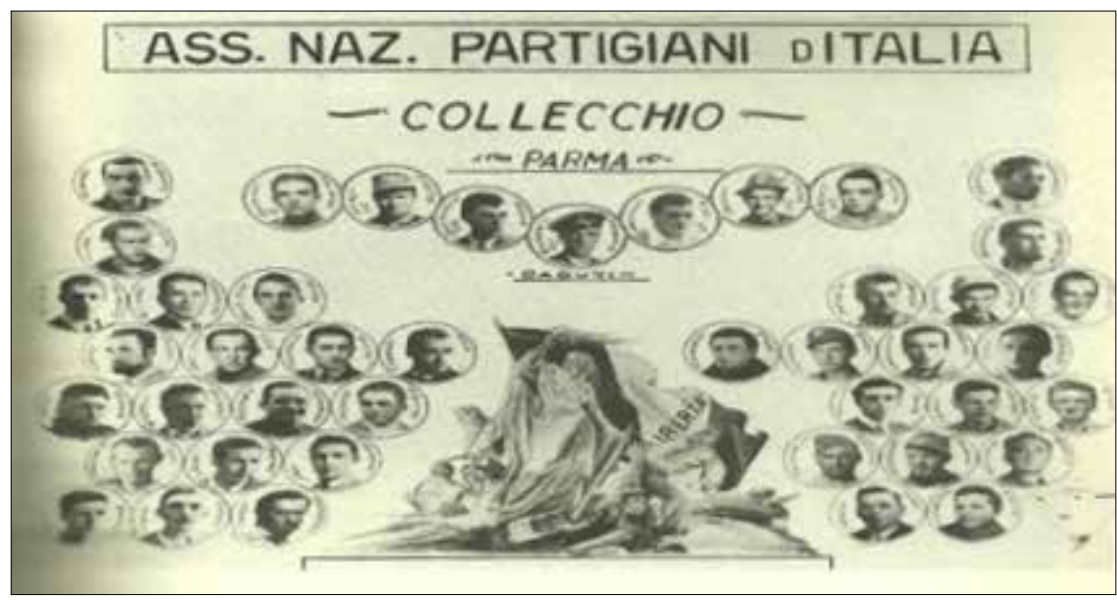

A sinistra dal basso, il terzo nella seconda riga è Giovanni Savi

Il prozio di mia madre all' età di 25 anni entrò a far parte dei partigiani e si nascondeva nei boschi o nelle montagne per combattere i tedeschi. Il suo nome di battaglia era "Baraca". [Lorenzo Giuberti]

Dopo l'otto settembre, il fratello maggiore [di mia nonna] [...], avendo paura di essere fatto prigioniero dalle truppe tedesche, pensò di fuggire, prendendo parte alla resistenza partigiana in Valceno, nella zona tra Fosio e Specchio [...] Una sera d'inverno, i Tedeschi stavano ispezionando la zona per trovare dei partigiani e giustiziarli. Se non fosse stato per un suo amico, che lo aveva fatto nascondere in un pagliaio, sarebbe stato fucilato insieme ad altri ragazzi che erano già stati catturati e uccisi nei pressi di Fornovo. [Thomas Porta]

In un paesino vicino a Ravarano, chiamato Lama, viveva una famiglia di mezzadri, i Bernini, i quali ospitavano nella loro casa i partigiani, essi scendevano stanchi dal nostro Appennino, la famiglia forniva loro cibo e munizioni. Qualche giorno prima della sera del 4 dicembre 1944 alcuni partigiani si incamminarono verso Corniglio, ma durante il tragitto, avendo avvistato i tedeschi, decisero di tornare indietro, verso casa Bernini. Il capo dei partigiani, un tipo magro e di statura 
normale con il pizzetto rosso, cominciò a girarsi tra le dita un proiettile calibro nove e ripeteva: "Il proiettile che deve uccidermi non è ancora stato fatto". Il suo nome di battaglia era "Zannarossa" (Bruno Ferrari), perché era il più deciso e attivo, non aveva paura di sfidare i Tedeschi. [Matilde Bottarelli]

A Collecchio la Liberazione giunse solo il 27 aprile, e in alcune zone limitrofe, tra Gaiano e Ozzano, fino all'ultimo giorno del mese si continuò ancora a combattere contro le truppe tedesche in ritirata, che tentavano di uscire dalla "sacca di Fornovo" [Battaglia 1983; Cosenza 1975; Delsante 1995]. Oltre ai partigiani e agli anglo-americani, a contribuire in modo significativo e definitivo all'affrancamento dall'occupazione nazifascista furono anche le forze armate brasiliane della Feb (Força Expedicionária Brasileira). Il ricordo del loro operato è ancora oggi molto presente sul piano della memoria e delle commemorazioni pubbliche, e ciò si riflette anche nelle considerazioni degli studenti:

L'intervento della Força Expedicionaria Brasileira (Feb), a sostegno della lotta per la libertà dall'occupazione tedesca, ha fatto "sbocciare" un'amicizia con un popolo tanto lontano... [Lorenzo Benassi]

I soldati della Feb che con scarsi mezzi sono riusciti a vincere numerose battaglie e a stupire il governo statunitense comunicando che "Il cobra ha fumato!" [Leonardo Giordani]

\section{Conclusioni. Didattica, memoria, consapevolezza}

Sul piano didattico, l'approccio all'insegnamento della storia adottato durante i laboratori, basato sulla convinzione che senza conoscenza, e senza una successiva riflessione, non sia possibile attivare un meccanismo del ricordo lontano da manifestazioni retoriche e semplificazioni, è risultato fertile, in quanto gli studenti si sono trovati a muoversi nel presente con maggiore consapevolezza. Sono divenuti protagonisti del processo che dall'apprendimento arriva alla comprensione e alla costruzione della memoria, attraversando la tappa, intermedia ed ineliminabile, dello studio e dell'interpretazione delle fonti e delle considerazioni critiche. Quello dell'Istituto è un approccio di educazione alla ricerca che può rappresentare - come in questo caso - un'educazione alla responsabilità del proprio muoversi nel mondo e nelle circostanze odierne. L'utilizzo di un simile metodo non intende tuttavia ridurre la rilevanza della dimensione della memoria nella società attuale: oggi più che mai il bisogno di ricordare è forte, ma la conoscenza arriva normalmente mediata da numerosi filtri, essendo venute a mancare, con il trascorrere del tempo, l'esperienza diretta o le testimonianze dei protagoni- 
sti. Si è dunque ritenuto necessario fare in modo che questa distanza divenga non un limite, ma un'opportunità: non un elemento di dispersione progressiva degli avvenimenti, bensì un veicolo per proporre una memoria epurata dalla retorica $\mathrm{e}$ dal coinvolgimento attivo nelle vicende, più di prima suffragata dalle fonti, dalla storiografia, e anche dalle testimonianze orali, ma attraverso intrecci e raffronti con altra documentazione. In questo senso e in questa direzione gli storici degli istituti, figure di mediazione tra l'accademia e la società, hanno un ruolo fondamentale nelle scuole.

Con le classi di Collecchio questo lavoro è stato effettuato e ciò ha permesso agli studenti di accorciare la distanza tra concetti astratti e situazioni realmente verificatesi sul territorio: in buona sostanza gli avvenimenti studiati, intrecciati con un lavoro sull'apparato documentale disponibile e sulla dimensione microstorica fornita dalle testimonianze, si sono fatti - per gli studenti - reali.

\section{Bibliografia}

Battaglia R., 1983, Storia della Resistenza italiana, Torino: Einaudi.

Cosenza E., 1975, La Sacca di Fornovo. Aprile 1945, Parma: Istituto storico della Resistenza per la Provincia di Parma.

Delsante U. (ed.), 1995, La guerra a Collecchio. Popolazione, partigiani ed eserciti di occupazione nel secondo conflitto mondiale, Collecchio: Amministrazione comunale di Collecchio. 